\title{
A Tree-profile Shape Ultra Wide Band Antenna for Chipless RFID Tags
}

\author{
A K M Zakir Hossain ${ }^{1}$, Nurulhalim Bin Hassim ${ }^{2}$, Jamil Abedalrahim Jamil Alsayaydeh ${ }^{3}$ \\ Centre for Telecommunication Research \& Innovation (CeTRI), Fakulti Teknologi Kejuruteraan Elektrik \& Elektronik (FTKEE) \\ Universiti Teknikal Malaysia Melaka (UTeM), Melaka \\ Mohammad Kamrul Hasan ${ }^{4}$ \\ Center for Cyber Security \\ Faculty of Information Science and Technology \\ The National University of Malaysia \\ Kuala Lumpur, Malaysia \\ Md. Rafiqul Islam ${ }^{5}$ \\ Department of Electrical and Computer Engineering \\ Faculty of Engineering \\ International Islamic University Malaysia \\ Gombak, Kuala Lumpur, Malaysia
}

\begin{abstract}
In this article, a new small size planar microstrip tree profile shaped Ultra-Wide Band (UWB) antenna with partial ground plane has been presented. The antenna is designed for chipless RFID tags that are working in UWB region. The operating frequency of the antenna is between $2.72 \mathrm{GHz}$ to 11.1 GHz which covers the entire UWB frequency band. The antenna exhibits comparatively high realized gain of $4.2 \mathrm{dBi}$ with respect to its small size of $27 \times 40 \mathrm{~mm}^{2}$ and have a gain to aperture ratio of 0.243 which is comparatively higher than other existing retransmission-based chipless RFID antennas. Another aspect of this antenna is its total efficiency which never goes below $80 \%$ throughout the entire bandwidth whereby it reaches as high as 96\% at 3.5GHz. This design will motivate the chipless RFID designers to produce small size and cost effective tags.
\end{abstract}

Keywords-Planar microstrip; UWB antenna; chipless RFID; realized gain; total efficiency

\section{INTRODUCTION}

Since the first application of the Radio Frequency IDentification (RFID) in WWII to distinguish the friendly airplanes from foe, the RFID has gone through many phases of development and elevated to a level of a dedicated and reliable technology for tagging and IDentification (IDing). In application such as livestock management, library management, retail shop, inventory management, toll collection and many others, the RFID system is Omni-present [1]. Based on the availability of on-board power supply on the RFID tags, the RFID can be classified into three classes i) Active (with battery onboard), ii) semi-active/passive RFID (with battery onboard but only to keep the memory chip alive and depends on the reader signal power for communication) and iii) passive RFID (has no battery and fully depends on the reader signal power to perform all functionality). Again, the passive type RFIDs can be sub-divided into two different kinds, a) chipped RFID and b) chipless RFID (CRFID). Among these kinds, the CRFID is inherently cheap due to the absence of the chip [2-3]. The preexisting barcode system is used widely in the industry. However, this system has many problems such as it is vulnerable to wear, tear and dent, tempering, low security and needs the line of sight (LOS) for detection. Whereas, the CRFID solves all these issues and is the only contender at the forefront to fully replace the barcode system. However, the CRFID still couldn't beat the cheapness of the barcode system. This is due to the comparatively bigger dimensions of the CRFID tag. The CRFID may or may not involve the antennas onboard. However, those CRFID systems that have no antennas on the tag (called backscatter and RCS base CRFID) suffers several issues such as crosstalk problem at the reader and lead to huge difficult to detect [4]. On the other hand, the CRFID with antennas on the tag (called retransmission-based CRFID) solves these issues by using the antenna polarization mismatch technique. It utilizes an antenna with horizontal polarization at the transmitter (Tx) part of the reader which is matched with the receiving $(\mathrm{Rx})$ antenna of the tag. Similarly, the tag's Tx antenna is vertically polarized and matched with the Rx antenna at the reader. This configuration imposes a 90-degree polarization mismatch between the $\mathrm{Rx}$ and Tx antennas of both reader and tag; and makes a theoretical zero crosstalk between them. However, the solution comes with a price of making the CRFID tag comparatively bigger than the backscatter/RCS based tag [1]. Fig 1 illustrates the basic working principle of a retransmission based CRFID system.

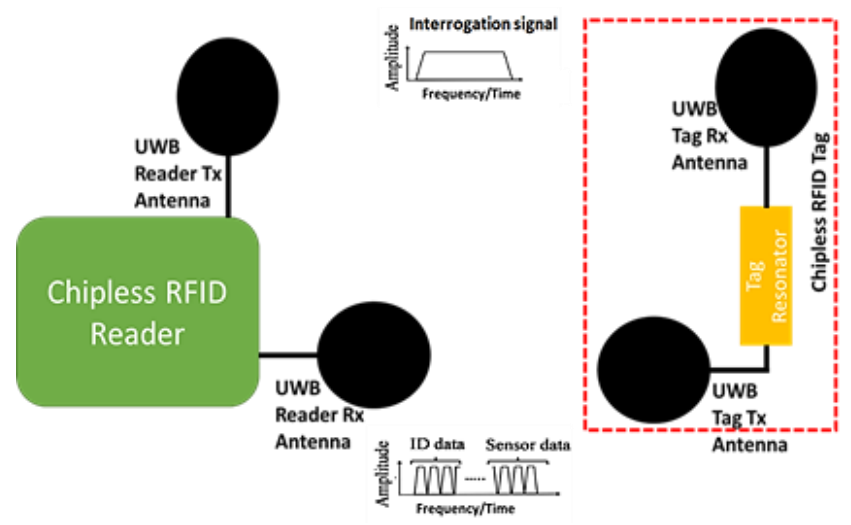

Fig. 1. The Chipless RFID System.

\footnotetext{
*Corresponding Author
} 
This article is organized as: II. Related work (where the current trends are addressed and benchmarked with this proposal). III. MTPS antenna design (in this section the design procedure is described and elaborated). IV. Results and Discussions (comprises the results and analysis related to the proposed design) and V. Conclusion (where the article is concluded and future recommendation has been made).

\section{RELATED WORKS}

One of the prominent retransmission-based CRFID has been proposed in [5] which has microstrip disc loaded monopole antennas (DLMA) with a large dimension of $60 \times$ $66 \mathrm{~mm}^{2}$ covering the entire bandwidth (BW) of $3.2 \mathrm{GHz}$ to $10.7 \mathrm{GHz}$ (defined UWB by FCC). Despite of the large dimension of that antenna, it only exhibits a low realized gain of $0 \mathrm{~dB}$. Another microstrip DLMA antenna has been proposed in [6] for a CRFID multi-state tag which has a large size of 60 $\times 90 \mathrm{~mm}^{2}$. However, no gain/directivity Vs frequency data has been presented in this work. The same DLMA structure has been proposed in [7], however, using the coplanar waveguide (CPW) feeding for the retransmission based CRFID system on the flexible substrate (Kapton film) to utilize it for liquid concentration sensing. The dimension of the proposed antenna is $40.6 \times 51.1 \mathrm{~mm}^{2}$, still, with a low gain of $0 \mathrm{dBi}$. To increase the gain of the antenna, in [4], the DLMA structure has been modified into an elliptical structure, using the same CPW feeding technique. The gain has been observed as $2 \mathrm{dBi}$, yet, in this particular proposal the actual size of the proposed antenna is missing. In [8], another proposal has been made by using the same CPW feed but the DLMA structure has been modified into a semi-circle structure. The proposed antenna has a comparatively small dimension of $35 \times 32 \mathrm{~mm}^{2}$. However, the authors have not presented any directivity/gain information in that particular work.

So far, the smallest antenna (a rectangular patch) has been proposed in [9] with a dimension of $23 \times 36 \mathrm{~mm}^{2}$ and it has a maximum gain of $2.45 \mathrm{dBi}$ for the retransmission-based CRFID tags. However, the gain to aperture (total area used by the antenna) ratio is still low at 0.23 . The same rectangular patch UWB antenna has been modified and proposed in [10] for a 3-state CRFID tag. The antenna exhibits a good gain of $4.9 \mathrm{dBi}$, yet, that is only due to the large dimension $(25 \times$ $74.62 \mathrm{~mm}^{2}$ ) of the antenna by achieving a gain to aperture ration of only 0.16 . Table I summarizes the complete scenario.

TABLE I. SUMMARY OF THE EXISTING WORKS

\begin{tabular}{|l|l|l|l|}
\hline References & $\begin{array}{l}\text { Antenna } \\
\text { Size }\left(\mathbf{m m}^{2}\right)\end{array}$ & $\begin{array}{l}\text { Gain } \\
(\mathbf{d B i}) /(\text { Linear})\end{array}$ & $\begin{array}{l}\text { Gain to Aperture } \\
\text { ratio (linear) }\end{array}$ \\
\hline$[1]$ & na & na & - \\
\hline$[4]$ & na & $2 / 1.58$ & - \\
\hline$[5]$ & $60 \times 66$ & $0 / 1$ & 0.025 \\
\hline$[6]$ & $60 \times 90$ & na & na \\
\hline$[7]$ & $40.6 \times 51.1$ & $0 / 1$ & 0.048 \\
\hline$[8]$ & $35 \times 32$ & na & na \\
\hline$[9]$ & $23 \times 36$ & $2.45 / 1.75$ & 0.23 \\
\hline$[10]$ & $25 \times 74.62$ & $4.9 / 3.01$ & 0.16 \\
\hline Proposed work & $\mathbf{2 7} \times \mathbf{4 0}$ & $\mathbf{4 . 2 / 2 . 6 3}$ & $\mathbf{0 . 2 4 3}$ \\
\hline
\end{tabular}

For all those aforementioned antennas, some have good gain but due to the large size and other have smaller dimensions, however, suffered a low gain for the chipless RFID system. In this proposed work, a microstrip tree-profile shape (MTPS) antenna has been proposed, designed and simulated for comparatively small size and higher realized gain to overcome these issues.

\section{MTPS ANTENNA DESIGN}

Fig 2 shows the proposed MTPS antenna structure for the CRFID tag. The antenna has been modeled on the dielectric substrate Rogers RT/ Duroid 5880. This substrate material has a relative permittivity/dielectric constant $\left(\varepsilon_{r}\right)$ of 2.2 , the loss tangent/dissipation factor $(\tan \delta)$ of 0.0009 and the dielectric height of $0.508 \mathrm{~mm}$. The antenna has front patch which looks like a tree profile shape and can be seen in the front view in Fig 1 . The MTPS patch is connected with a microstrip transmission feed line of $50 \Omega$ to the modeled SMA connector. At the back view of the proposed structure, it can be seen that the antenna ground plane has a partial ground plane (PGP) to promote the structure to a UWB antenna geometry.

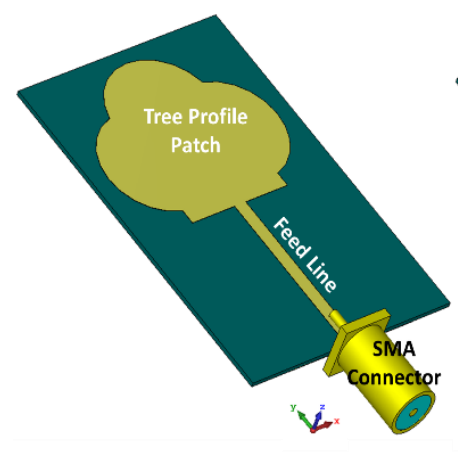

Front View

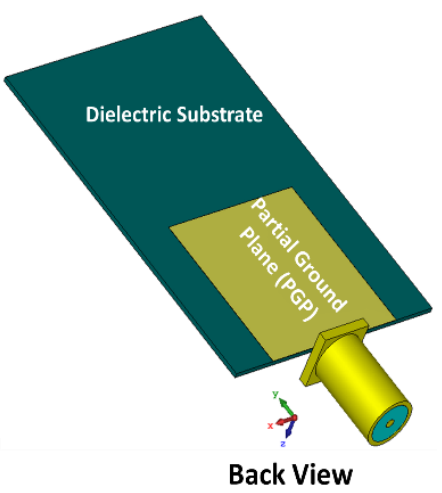

Back View
Fig. 2. The Geometry of the Proposed Antenna.
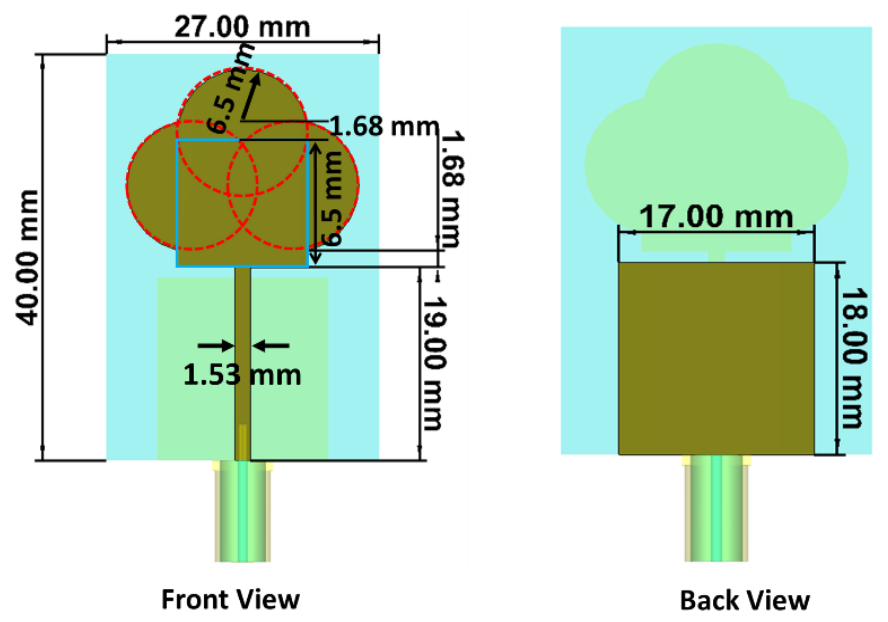

Fig. 3. The Exact Dimensions of the Proposed Antenna.

Fig 3 reveals the exact dimension of the different segments of the proposed MTPS antenna. It can be seen that to create a tree-profile shape it constitutes three differently placed circular patches along with a square patch. All circular patches have the same radius of $6.5 \mathrm{~mm}$ whereby the square patch has the side 
lengths same as the radius of the circular patch. The radius location of the center circular patch is at the bisection point of the top side of the square but it is $1.68 \mathrm{~mm}$ higher from that point towards Y-axis. Similarly, the centers of the other two circular patches are also on the two corresponding sides of the square but $1.68 \mathrm{~mm}$ higher from the bisection point of them towards Y-axis. All these four different patches are merged together to form a tree-profile shape. Furthermore, a TL $(50 \Omega)$ feed having width of $1.53 \mathrm{~mm}$ has been connected with the tree-profile shaped patch that has a length of $19 \mathrm{~mm}$ to create a MTPS antenna with a final dimension of $27 \times 40 \mathrm{~mm}^{2}$. From the back view in Fig 3, it can be seen that the PGP has a length and width of $18 \mathrm{~mm}$ and $17 \mathrm{~mm}$ respectively.

These dimensions are in fact approximated and later optimized with the help of equation (1) - (9) [11-14].

$$
\begin{aligned}
& W=\frac{c_{0}}{2 f_{c} \sqrt{\frac{\left(\varepsilon_{r}+1\right)}{2}}} \\
& \varepsilon_{e}=\frac{\varepsilon_{r}+1}{2}+\frac{\varepsilon_{r}-1}{2}\left[1+12 \frac{h}{W_{p}}\right]^{-\frac{1}{2}} \\
& L_{e}=\frac{C_{0}}{2 f_{c} \sqrt{\varepsilon_{e}}} \\
& \Delta L=0.412 h \frac{\left(\varepsilon_{e}+0.3\right)\left(\frac{W_{k}}{h}+0.264\right)}{\left(\varepsilon_{e}-0.258\right)\left(\frac{W_{k}}{h}+0.8\right)} \\
& L=L_{e}-2 \Delta L \\
& L_{g}=6 h+L_{k} \\
& W_{g}=W_{g}=6 h+W_{k} \\
& r=\frac{F}{\left\{1+\frac{2 h}{\pi \varepsilon_{r} F}\left[\ln \left(\frac{\pi F}{2 h}\right)+1.7726\right]\right\}^{\frac{1}{2}}} \\
& F=\frac{8.791 \times 10^{9}}{f_{c} \sqrt{\varepsilon_{r}}}
\end{aligned}
$$

Where, the $\mathrm{L}$ and the $\mathrm{W}$ are the length and the width of the square patch for the MTPS antenna respectively. $\varepsilon_{e}$ and $\varepsilon_{r}$ are the effective and relative dielectric constant respectively; and $C_{0}$ is the speed of the light. $\mathrm{W}_{\mathrm{g}}$ and $\mathrm{L}_{\mathrm{g}}$ are the approximated width and length of the PGP respectively, before optimization. Furthermore, $r$ is the approximated radius of those three circles for the MTPS antenna patch. The approximation has been done by the above stated equations in the beginning. Later, in the CST MWS 2020 simulator, the optimization has been done to achieve the desired BW for the antenna and the final dimensions for the designed MTPS antenna are shown in Fig 3. In addition, the conductors in the design (patch, TL and ground plane) have been modeled as perfect electric conductor (PEC) in the simulator.

\section{RESUlTS AND DISCUSSIONS}

Fig 4 illustrates three important responses of the designed MTPS antenna; reference impedance, S-parameter and voltage standing wave ratio (VSWR). For any antenna design, the first thing to check is the reference impedance. Since, the antenna will be connected to a system/connector which would have a specific characteristic impedance. In this case, the antenna is intended to be connected with a $50 \Omega$ system. So, the antenna reference impedance has to be as close as to $50 \Omega$ for a good matching and, less reflection and return loss. Fig 4 (a) shows that the antenna reference impedance is exactly $50 \Omega$ throughout the span of $1-12 \mathrm{GHz}$. Thus, the preliminary design integrity check has been fulfilled.

The next step is to check the $\mathrm{S}$-parameter $\left(\mathrm{S}_{11}\right)$ response of this antenna to see the preliminary $-10 \mathrm{~dB}$ BW. From Fig 4 (b) it can be observed that the $-10 \mathrm{~dB}$ BW for the MTPS antenna starts at $2.75 \mathrm{GHz}$ and ends at $10.95 \mathrm{GHz}$. So, based on this response a $\mathrm{BW}$ of $8.2 \mathrm{GHz}$ is achieved which covers the whole FCC defined UWB frequency. However, to get a confirmation on the actual operating frequency of the antenna, it is necessary to check the VSWR as well. It is known form the theory that a perfect/ideal VSWR for any passive microwave TL is 1. Again, as long as the VSWR value stays between 1-2, the antenna can be operate-able on those frequency band(s). From Fig 4 (c), it can be seen that the antenna has a VSWR BW of $2.72 \mathrm{GHz}$ to 11.1 GHz. This actually confirms the original working BW of $8.38 \mathrm{GHz}$ for this designed antenna. Fig 5 illustrates the outband (at $2 \mathrm{GHz}$ ) and in-band (at $6.5 \mathrm{GHz}$ ) surface current distribution/accumulation of the MTPS antenna. It is seen that at $2 \mathrm{GHz}$ most of the surface current accumulates on the feed line and does not reach on the antenna patch for radiation which implies the antenna cannot radiate good at that frequency. Whereby, at $6.5 \mathrm{GHz}$ it is clearly visible that the current accumulation reaches on the patch of the MTPS antenna for radiation. This is another justification on the quality of the design.

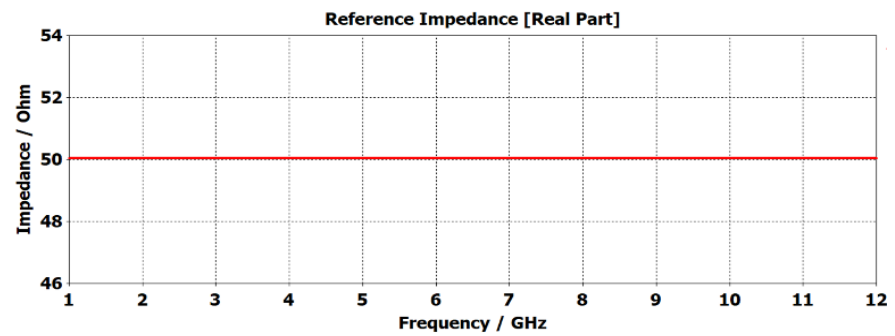

(a) Reference Impedance.

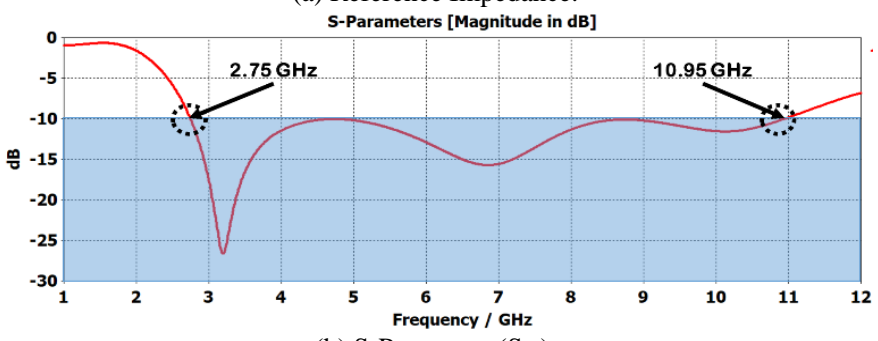

(b) S-Parameter $\left(\mathrm{S}_{11}\right)$

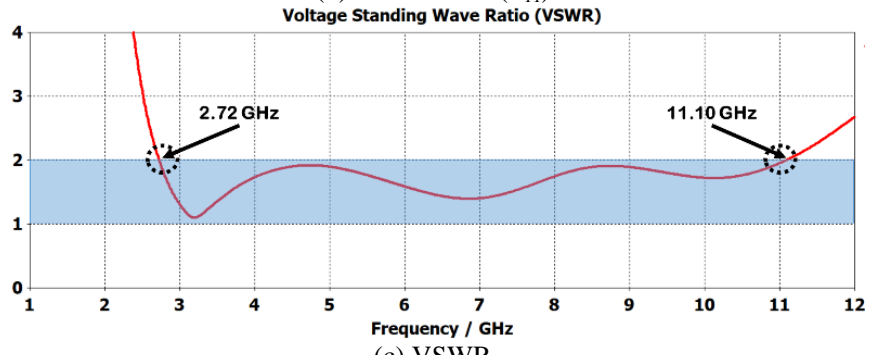

(c) VSWR.

Fig. 4. The (a) Reference Impedance, the (b) S-Parameter (S11) and the (c) VSWR of the Antenna. 

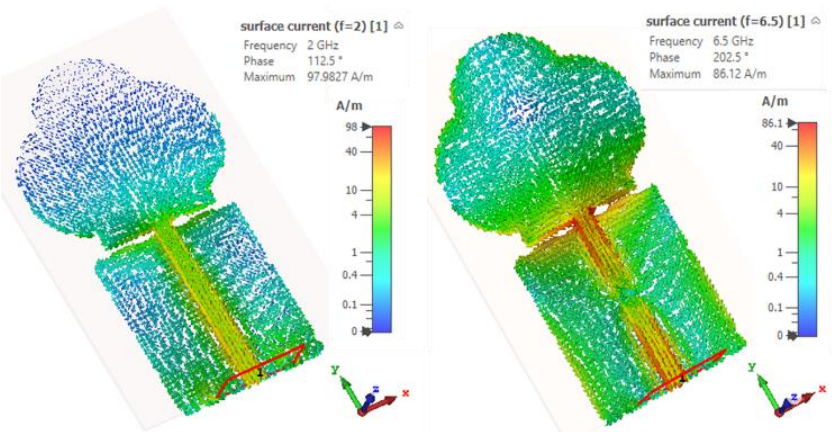

Fig. 5. The Surface Current Distribution at $2 \mathrm{GHz}$ and $6.5 \mathrm{GHz}$.

Next, it is necessary to investigate the farfield radiation capabilities of the antenna. Fig 6 comprises the 3-D and polar responses of the farfield radiation pattern of the MTPS antenna at $3.2 \mathrm{GHz}, 6.5 \mathrm{GHz}$ and $10.7 \mathrm{GHz}$. Fig 6 (a), (c) and (e) illustrate the 3-D patterns for at $3.2 \mathrm{GHz}, 6.5 \mathrm{GHz}$ and 10.7 $\mathrm{GHz}$ respectively. Whereby, Fig 6 (b), (d) and (f) include the polar form of the horizontal (H-) and the elevation (E-) plane pattern at those three frequencies respectively. From the 3.2 $\mathrm{GHz}$ response it can be seen that the 3-D pattern is a semi omni-directional pattern where the H-plane is circular and the E-plane is bidirectional in nature. With the increases in the frequency, the farfield pattern becomes more directional as can be seen in Fig 6 (c) and (d). Here, it is observed that the antenna becomes completely bidirectional having pointing lobes at the front and back side of the antenna. The rest of the direction of the antenna, it tends to decrease the intensity of the radiation. From the E- and the $\mathrm{H}$-plane polar representation also justifies the bidirectional nature of the antenna at $6.5 \mathrm{GHz}$. The response at $10.7 \mathrm{GHz}$ showing that the antenna no longer radiates at the front and back of the antenna structure, rather, it scans the $45^{\circ}$ angles from the front and back axis and also radiates prominently at the $\mathrm{Z}$-axis direction. Lastly, it is important also to observe the antenna efficiencies and the realized gain of the antenna. Fig 7 discloses the responses of efficiencies and realized gain vs frequency.

From Fig 7(a), it is realized that both of the efficiency responses are good. The radiation efficiency of the antenna is constant throughout the entire BW between $92 \%$ and $99 \%$. Whereby, the total efficiency of the antenna never goes below $80 \%$ starting from $2.7 \mathrm{GHz}$ to $11 \mathrm{GHz}$. Furthermore, it reaches as high as $96 \%$ at $3.5 \mathrm{GHz}$ and keeps itself nominally constant around $90 \%$ which indicates good quality of the design. Fig 7 (b) comprises the realized gain Vs frequency result of the designed MTPS antenna. It can be seen that the maximum realized gain is $4.2 \mathrm{dBi}$ at $6.7 \mathrm{GHz}$. The gain at the beginning of the bandwidth starts with a value of $2 \mathrm{dBi}$ and reaches at its peak at $6.7 \mathrm{GHz}$ and starts falling until $8.8 \mathrm{GHz}$ where the lowest gain of $0.45 \mathrm{dBi}$ can be observed. The gain starts showing rising trends after that point and cross-passes through the working BW with the same trend. All these results presented in this section justify the quality of the proposed MTPS antenna for the retransmission-based CRFID tags and the reader as well.

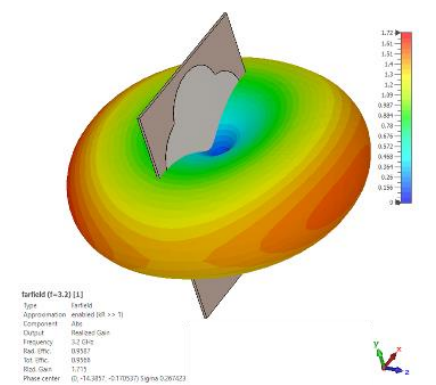

(a) $3.2 \mathrm{GHz}$

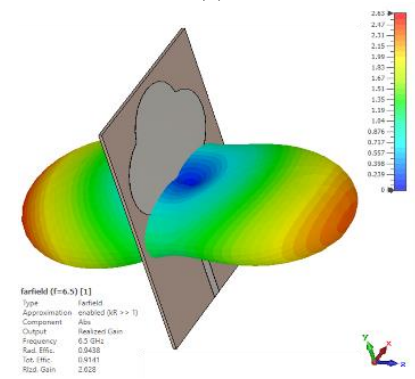

(c) $6.5 \mathrm{GHz}$

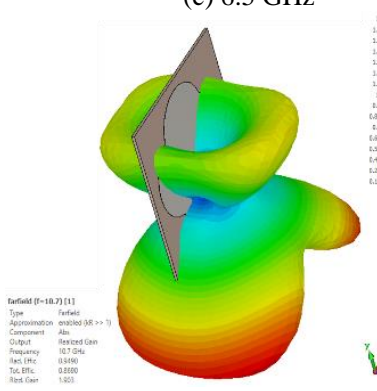

(e) $10.7 \mathrm{GHz}$

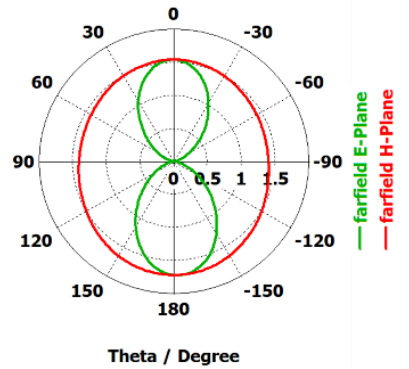

(b) $3.2 \mathrm{GHz}$
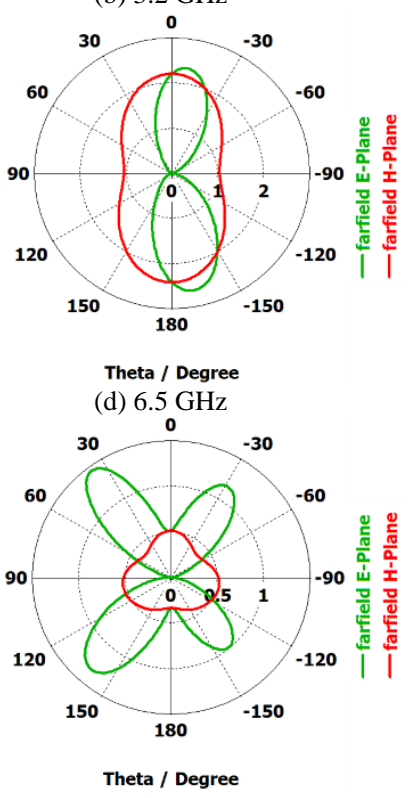

(f) $10.7 \mathrm{GHz}$
Theta / Degree

Fig. 6. The 3-D and 2-D (Polar) Representation of the Farfield Radiation Pattern of the MTPS Antenna.

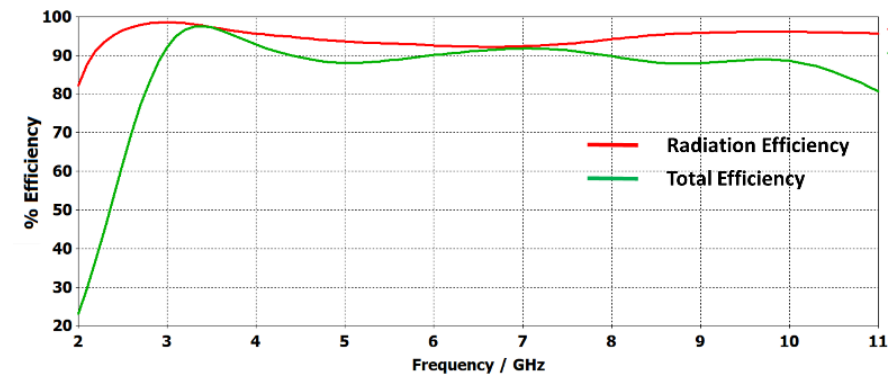

(a) Radiation and Total Efficiency.

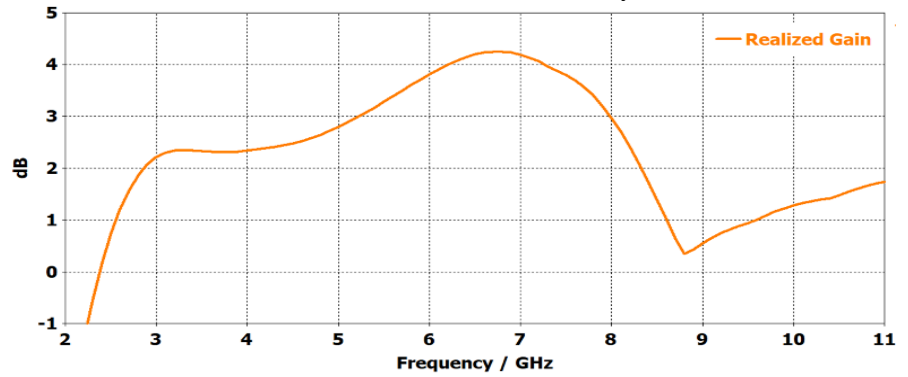

(b) Realized Gain.

Fig. 7. The (a) Antenna Efficiencies and the (b) Realized Gain Vs Frequency of the Antenna. 


\section{CONCLUSION}

A planar microstrip tree profile shaped (MTPS) UWB antenna for retransmission-based CRFID has been designed and analyzed. The antenna has been modeled in CST MWS and realized on Rogers RT/Duroid 5880. The antenna works between $2.72 \mathrm{GHz}$ to $11.1 \mathrm{GHz}$ which covers the whole UWB frequency allocation. The antenna is small in size, exhibits a good maximum realized gain of $4.2 \mathrm{dBi}$ and has a gain to aperture ratio of 0.243 which is higher than other existing proposed antennas for retransmission-based CRFID applications. The farfield radiation pattern and the efficiencies also indicate the good quality of the design. This proposed antenna will motivate the CRFID researchers to design a comparatively smaller, efficient and cost effective CRFID tags. The future aspect of this work is to implement the proposed antenna with a full CRFID system to assess the capability of this design.

\section{ACKNOWLEDGMENT}

This work has been funded by the center for research and innovation management (CRIM), University Teknikal Malaysia Melaka (UTeM).

\section{REFERENCES}

[1] Hossain, A. K. M. Z., Ibrahimy, M. I., Motakabber, S. M. A., Azam, S. M. K., \& Islam, M. S. (2021). Multi-resonator application on size reduction for retransmission-based chipless RFID tag. Electronics Letters, 57(1), 26-29.

[2] Hossain, A. K. M. Z., Motakabber, S. M. A., \& Ibrahimy, M. I. (2015). Microstrip spiral resonator for the UWB chipless RFID tag. In Progress in Systems Engineering (pp. 355-358). Springer, Cham.

[3] Hossain, A. K., Ibrahimy, M. I., \& Motakabber, S. M. (2014). Spiral resonator for ultra wide band chipless RFID tag. In 2014 International Conference on Computer and Communication Engineering (pp. 281283). IEEE.

[4] Bhuiyan, M. S., \& Karmakar, N. C. (2014). An efficient coplanar retransmission type chipless RFID tag based on dual-band McSrr. Progress In Electromagnetics Research, 54, 133-141. doi:10.2528/PIERC14061403.
[5] Preradovic, S., Balbin, I., Karmakar, N. C., \& Swiegers, G. F. (2009). Multiresonator-based chipless RFID system for low-cost item tracking. IEEE Transactions on Microwave Theory and Techniques, 57(5), 14111419. doi: 10.1109/TMTT.2009.2017323.

[6] Majidifar, S., Ahmadi, A., Sadeghi-Fathabadi, O., \& Ahmadi, M. (2015). A novel phase coding method in chipless RFID systems. AEUInternational Journal of Electronics and Communications, 69(7), 974980. doi:10.1016/j.aeue.2015.02.013.

[7] Li, Z., \& Bhadra, S. (2019). A 3-bit fully inkjet-printed flexible chipless RFID for wireless concentration measurements of liquid solutions. Sensors and Actuators A: Physical, 299, 111581. doi:10.1016/j.sna.2019.111581.

[8] Weng, Y. F., Cheung, S. W., Yuk, T. I., \& Liu, L. (2013). Design of chipless UWB RFID system using a CPW multi-resonator. IEEE Antennas and Propagation Magazine, 55(1), 13-31. doi: 10.1109/MAP.2013.6474480.

[9] Ma, Z. H., Yang, J. H., Chen, C. C., \& Yang, C. F. (2018). A retransmitted chipless tag using CSRR coupled structure. Microsystem Technologies, 24(10), 4373-4382. doi:10.1007/s00542-018-3836-z.

[10] Abdulkawi, W. M., \& Sheta, A. A. (2020). High coding capacity chipless radiofrequency identification tags. Microwave and Optical Technology Letters, 62(2), 592-599. doi:10.1002/mop.32057.

[11] Islam, M. S., Ibrahimy, M. I., Motakabber, S. M. A., \& Hossain, A. Z. (2018, September). A Rectangular Inset-Fed Patch Antenna with Defected Ground Structure for ISM Band. In 2018 7th International Conference on Computer and Communication Engineering (ICCCE) (pp. 104-108). IEEE. doi:10.1109/ICCCE.2018.8539260.

[12] Islam, M. S., Ibrahimy, M. I., Motakabber, S. M. A., Hossain, A. Z., \& Azam, S. K. (2019). Microstrip patch antenna with defected ground structure for biomedical application. Bulletin of Electrical Engineering and Informatics, 8(2), 586-595. doi:10.11591/eei.v8i2.1495.

[13] Azam, S. M. K., Islam, M. S., Hossain, A. K. M. Z., \& Othman, M. (2020). Monopole antenna on transparent substrate and rectifier for energy harvesting applications in 5G. International Journal of Advanced Computer Science and Applications, 11(8), 84-89. doi:10.14569/IJACSA.2020.0110812.

[14] Zakir Hossain, A. K. M., Hassim, N. B., Kayser Azam, S. M., Islam, M. S., \& Hasan, M. K. (2020). A planar antenna on flexible substrate for future $5 \mathrm{~g}$ energy harvesting in malaysia. International Journal of Advanced Computer Science and Applications, 11(10), 151-155. doi:10.14569/IJACSA.2020.0111020. 